\title{
BMJ Open Patient-tailored triage decisions by anaesthesiologist-staffed prehospital critical care teams: a retrospective descriptive study
}

Morten Langfeldt Friberg, ${ }^{1,2}$ Leif Rognås ${ }^{2}$

To cite: Friberg ML, Rognås L. Patient-tailored triage decisions by anaesthesiologiststaffed prehospital critical care teams: a retrospective descriptive study. BMJ Open 2018:8:e019813. doi:10.1136/ bmjopen-2017-019813

- Prepublication history for this paper is available online. To view these files, please visit the journal online (http://dx.doi org/10.1136/bmjopen-2017019813).

Received 26 September 2017 Revised 9 May 2018 Accepted 13 June 2018
Check for updates

(c) Author(s) (or their employer(s)) 2018. Re-use permitted under CC BY-NC. No commercial re-use. See rights and permissions. Published by BMJ.

${ }^{1}$ Department of Thoracic Medicine, Haukeland University Hospital, Bergen, Norway ${ }^{2}$ The Pre-hospital Critical Care Service in Aarhus, Department of Pre-hospital Critical Care Service, Pre-hospital Medical Services, Central Denmark Region, Aarhus, Denmark

Correspondence to Dr Morten Langfeldt Friberg; morten.friberg@gmail.com

\section{ABSTRACT}

Objectives The primary objective was to estimate the incidence of patients in the Central Denmark Region triaged to bypass the local emergency department without being part of a predefined fast-track protocol. The secondary objective was to describe these triage decisions in more detail with regard to the most common diagnoses, incidence of direct referral sorted by the prehospital critical care team (PHCCT) and the destination hospital.

Design Retrospective descriptive study.

Setting and participants The emergency medical service in the Central Denmark Region primarily consists of emergency medical technician (EMT)staffed ambulances and anaesthesiologist-EMT-staffed PHCCTs. Patients treated by the nine ground-based PHCCTs in the region constituted the study population. The inclusion criteria were all patients treated by the PHCCTs during 2013 and 2014. The exclusion criteria were interhospital transfers, and patients with STsegment elevation myocardial infarction, stroke or were in active labour.

Endpoints Incidence of prehospital critical care anaesthesiologist-initiated direct referral, prehospital tentative diagnoses and transport destination. Results During the study period, the PHCCTs treated 39396 patients and diverted $989(2.5 \%)$ patients not covered by a predefined fast-track protocol to a specialised hospital department. 'Resuscitated from cardiac arrest' $(n=143)$, 'treatment and observations following road traffic accident' $(\mathrm{n}=105)$ and 'observation and treatment for an unspecified disease/condition' $(\mathrm{n}=78)$ were the most common prehospital tentative diagnoses, accounting for $33.0 \%$ of all diverted patients. In total, 943 (95.3\%) of the PHCCT-diverted patients were diverted to a department at Aarhus University Hospital.

Conclusion Our results demonstrate that in 1 out of 40 patient contacts, the anaesthesiologist-staffed PHCCTs in the Central Denmark Region divert critically ill and injured patients directly to a specialised hospital department, bypassing local emergency departments and potentially reducing time to definitive care for these patients. There may be a potential for increased referral of patients with no predefined fast-track directly to specialised departments in the Central Denmark Region.

\section{Strengths and limitations of this study}

- This is the first study to assess prehospital critical care team (PHCCT)-initiated direct referral to specialised care.

- The study is based on a comprehensive data set containing all prehospital emergency patients seen by the PHCCTs of the Central Denmark Region during a 2-year period.

- The retrospective design combined with our data set with all patients anonymised does not allow analysis of the accuracy of triage or comparison of outcomes, such as mortality and morbidity, for emergency patients referred directly to specialised department with patients referred to the nearest emergency department.

\section{BACKGROUND}

Many countries in Europe and Australasia use two-tiered emergency medical systems (EMS) with physician-paramedic prehospital teams, commonly staffed with anaesthesiologists with additional training in prehospital critical care. These prehospital critical care teams (PHCCTS) are often selectively deployed by helicopter or land-based rapid response vehicles to patients with time-critical conditions that may benefit from immediate diagnostic or therapeutic interventions provided by the PHCCT. ${ }^{12}$ In addition, the PHCCT may have the opportunity to triage and direct patients directly to specialised care, bypassing for instance local emergency departments (EDs).

There is solid evidence in favour of prehospital triage for time-critical conditions, for example, ST-segment elevation myocardial infarction $\left(\right.$ STEMI $\left.^{3-5}\right)$ and stroke $^{6-8}$. A recent systematic review found that in patients with STEMI, prehospital destination triage based on the transmission of 12-lead ECG with telemedicine reduces time to treatment by approximately $50 \% .{ }^{9}$ Survival and good functional outcome after stroke are 
inversely related to time from onset of symptoms to reperfusion. ${ }^{10}{ }^{11}$ In the Central Denmark Region, predefined fast-track protocols route patients with STEMI or stroke, as well as women in labour and children under the age of 15, to specific departments rather than local EDs. The EMS includes nine rapid response vehicles staffed with a prehospital critical care anaesthesiologist and a specially trained emergency medical technician (EMT) or paramedic. These PHCCTs initiate advanced prehospital diagnostic and therapeutic procedures and triage critically ill and injured patients to the most appropriate treatment facility. ${ }^{2}{ }^{12-18}$ The PHCCTs may decide to transport patients with no predefined fast-track directly to a specialised department, bypassing local EDs. This management protocol represents an attempt to reduce the delay of vital diagnostic and therapeutic procedures, hence potentially improving patient outcome. ${ }^{19}$ In these patients, critical decision-making replaces standardised criteria for fast-tracking.

International research suggests that implementing regional trauma systems may improve prehospital triage and thereby potentially reduce mortality and morbidity. ${ }^{20-26}$ We have been unable to identify studies reporting similar data from unselected patient populations.

The extent to which the PHCCTs in the Central Denmark Region choose to bypass local EDs in patients not included in a predefined fast-track protocol is not known.

Our primary objective was to estimate the incidence of patients in the Central Denmark Region triaged to bypass the local ED who were not part of a predefined fast-track protocol. Our secondary objective was to describe these triage decisions in more detail with regard to the most common diagnoses, incidence of direct referral sorted by the PHCCT and the destination hospital.

\section{MATERIALS AND METHODS}

\section{Study design}

We conducted a retrospective descriptive study.

\section{Setting}

The Central Denmark Region includes both urban and rural areas, covering $13005 \mathrm{~km}^{2}$ with a population of 1.282 million, which is equivalent to 98.6 inhabitants per square kilometre as of 31 December $2014 .{ }^{27}$ Medical dispatch in the Central Denmark Region has been described elsewhere. ${ }^{28} 29$

The Central Denmark Region has a two-tiered EMS system, wherein the first tier consists of 64 ground ambulances staffed with EMTs on an intermediate or paramedic level (EMT-I and EMT-P, respectively). The second tier consists of 10 PHCCTs. Rapid response vehicles deploy nine of the PHCCTs, and the tenth team staffs a helicopter emergency medical service (HEMS). The most rural parts have an additional intermediate tier with three rapid response vehicles staffed with nurse anaesthetists. ${ }^{12}$
Aarhus University Hospital is the main referral centre in the Central Denmark Region offering secondary and tertiary levels of care, and it is the only hospital offering all specialties and relevant subspecialties. It is also the major trauma centre for the region. Further information can be found at the hospital website. ${ }^{30}$ District general hospitals offer secondary-level healthcare services and are comparable in composition with that of many district general hospitals in the UK.

\section{Participants}

The inclusion criteria were all patients treated by the ground-based PHCCTs.

The exclusion criteria were interhospital transfers, patients with STEMI, patients with stroke and women in labour. Data from patients primarily treated by the HEMS of the Central Denmark Region were excluded from this study given that the ground-based EMS and the HEMS use different patient journal systems. However, all PHCCT-diverted patients initially seen by the PHCCTs but subsequently transported by HEMS directly to specialised department were included in our study.

\section{Variables and endpoints}

We searched the regional prehospital database for patients fulfilling our search criteria. Please see our data set, available in the Figshare Repository (DOI:10.6084/ m9.figshare.5852967), for a comprehensive overview.

The following was our primary endpoint:

- Number and proportion of PHCCT-diverted patients. The following were our secondary endpoints:

- Number and proportion of PHCCT-diverted patients among patients treated by the different PHCCTs.

- Number and proportion of PHCCT-diverted patients from the PHCCTs located less than $70 \mathrm{~km}$ from Aarhus University Hospital.

- Number and proportion of PHCCT-diverted patients from the PHCCTs located greater than $70 \mathrm{~km}$ from Aarhus University Hospital.

- Prehospital tentative diagnosis.

- Receiving hospital.

\section{Data sources and data collection}

We retrospectively searched the regional prehospital critical care database for all relevant patient contacts from 1 January 2013 until 31 December 2014. We performed an exhaustive overview of all possible prehospital diagnoses and all possible receiving hospitals for each of the Central Denmark Region's PHCGTs. For each PHCCT, we identified all patient transports where the combination of diagnoses and receiving hospital represented a diversion rather than a transport to the nearest ED while not being part of a predefined fast-track. Finally, we verified the eligibility through crosschecking the identified relevant missions with our exclusion criteria, as well as excluding all patients admitted at the nearest hospital.

\section{Study size}

Sample size and power calculations were not performed. 
Table 1 Number and percentage of PHCCT-diverted patients sorted according to PHCCT

\begin{tabular}{lcc}
\hline PHCCT & $\mathbf{n}(\%)$ & $\begin{array}{l}\text { Total number of } \\
\text { patients }\end{array}$ \\
\hline Grenaa & $172(6.12)$ & 2809 \\
\hline Silkeborg & $180(4.87)$ & 3695 \\
Horsens & $246(4.16)$ & 5920 \\
Randers & $155(3.01)$ & 5141 \\
Lemvig & $26(1.80)$ & 1446 \\
\hline Holstebro & $42(1.51)$ & 2774 \\
Viborg & $60(1.47)$ & 4088 \\
Herning & $55(1.43)$ & 3833 \\
Aarhus & $53(0.55)$ & 9690 \\
\hline Total (\%) & $989(\mathbf{2 . 5 1 )}$ & 39396 \\
\hline
\end{tabular}

$\%$, percentage of PHCCT-diverted patients out of all PHCCT patient contacts in each PHCCT; $n$, number of PHCCT-diverted patients; total number of patients, total number of PHCCT patient contacts in each specific PHCCT.

PHCCT, prehospital critical care team. The sum of all PHCCTdiverted patients, the sum of all PHCCT patient contacts and the overall percentage of PHCCT-diverted patients are marked with bold.

\section{Statistical methods}

We used descriptive statistics.

\section{Patient and public involvement}

No patients were involved in the development and design of the study.

\section{RESULTS}

\section{Overall study population}

The total number of PHCCT contacts was 39 396. In total, $6192(15.7 \%)$ were treated on scene only. Another 1938 (4.9\%) were declared dead, and $31266(79.4 \%)$ were admitted to the hospital.

\section{Total number and proportion of PHCCT-diverted patients}

The PHCCTs diverted 989 (2.5\%) patients who were not in active labour or did not experience a STEMI or a stroke directly to a specialised hospital department.

\section{Distribution of PHCCT-diverted patients sorted by PHCCT}

The number and proportion of PHCCT-diverted patients sorted by the different PHCCTs are presented in table 1 .

\section{Comparison of the number and proportion of PHCCT-diverted patients from PHCCTs at a distance more than and less than 70 km from Aarhus University Hospital}

The proportion of PHCCT-diverted patients sorted by PHCCT is on average 2.9 times lower in those PHCCTs with a travel distance of $75-150 \mathrm{~km}$ to Aarhus University Hospital compared with teams located $40-65 \mathrm{~km}$ away from Aarhus University Hospital (table 1 and figure 1).
Distribution of PHCCT-diverted patients sorted by prehospital diagnosis

The 10 most frequently used prehospital diagnoses among the PHCCT-diverted patients are provided in table 2. The diagnoses 'resuscitated from cardiac arrest' $(\mathrm{n}=143)$, 'treatment and observations following road traffic accident' $(\mathrm{n}=105)$ and 'observation and treatment for not specified disease/condition' $(\mathrm{n}=78)$ were the most common, collectively accounting for $33.0 \%$ of all PHCCT-diverted patients. Among these three diagnoses, all patients except one were diverted to Aarhus University Hospital.

\section{Distribution of PHCCT-diverted patients sorted by receiving hospital}

The distribution of PHCCT-diverted patients sorted by receiving hospital is presented in table 3 . The majority of PHCCT-diverted patients were diverted directly to a specialised department at Aarhus University Hospital. Of the 15 PHCCT-diverted patients admitted at Rigshospitalet in Copenhagen, 13 patients had burn injuries, 1 had multiple trauma and 1 was assigned the diagnosis 'observation and treatment following other injury'. The four university hospitals included in table 3 represent the Central, the North, the Southern, the Zealand and the Capital Region, listed accordingly. Rigshospitalet is the national tertiary referral centre for severe burns ( $>15 \%$ of body surface in adults and $>10 \%$ in children), and Odense University Hospital is the national tertiary referral centre for reimplantation of amputated extremities. All 14 PHCCT-diverted patients admitted at Viborg Regional Hospital shared the diagnosis of 'dissecting/ ruptured aortic aneurysm'.

\section{DISCUSSION}

To our knowledge, this is the first detailed description of patient-tailored triage by PHCCTs.

\section{Total number and proportion of PHCCT-diverted patients}

We were not able to identify any similar studies providing a basis for comparison of the direct referral rate. One might speculate that there may be an unexploited potential for triage of patients directly to specialised departments in the Central Denmark Region. It is essential that critical decision-making, including triage, is continuously optimised. The consequence of substandard triage may be increased time to definitive care and potentially increased mortality and morbidity and reduced patient safety.

\section{Number and proportion of PHCCT-diverted patients sorted by PHCCT}

The proportion of PHCCT-diverted patients ranges from $0.6 \%$ to $6.1 \%$ in the different PHCCTs of the Central Denmark Region. The geography of the Central Denmark Region and differences in demography, infrastructure and resources at the local hospitals are possible factors 


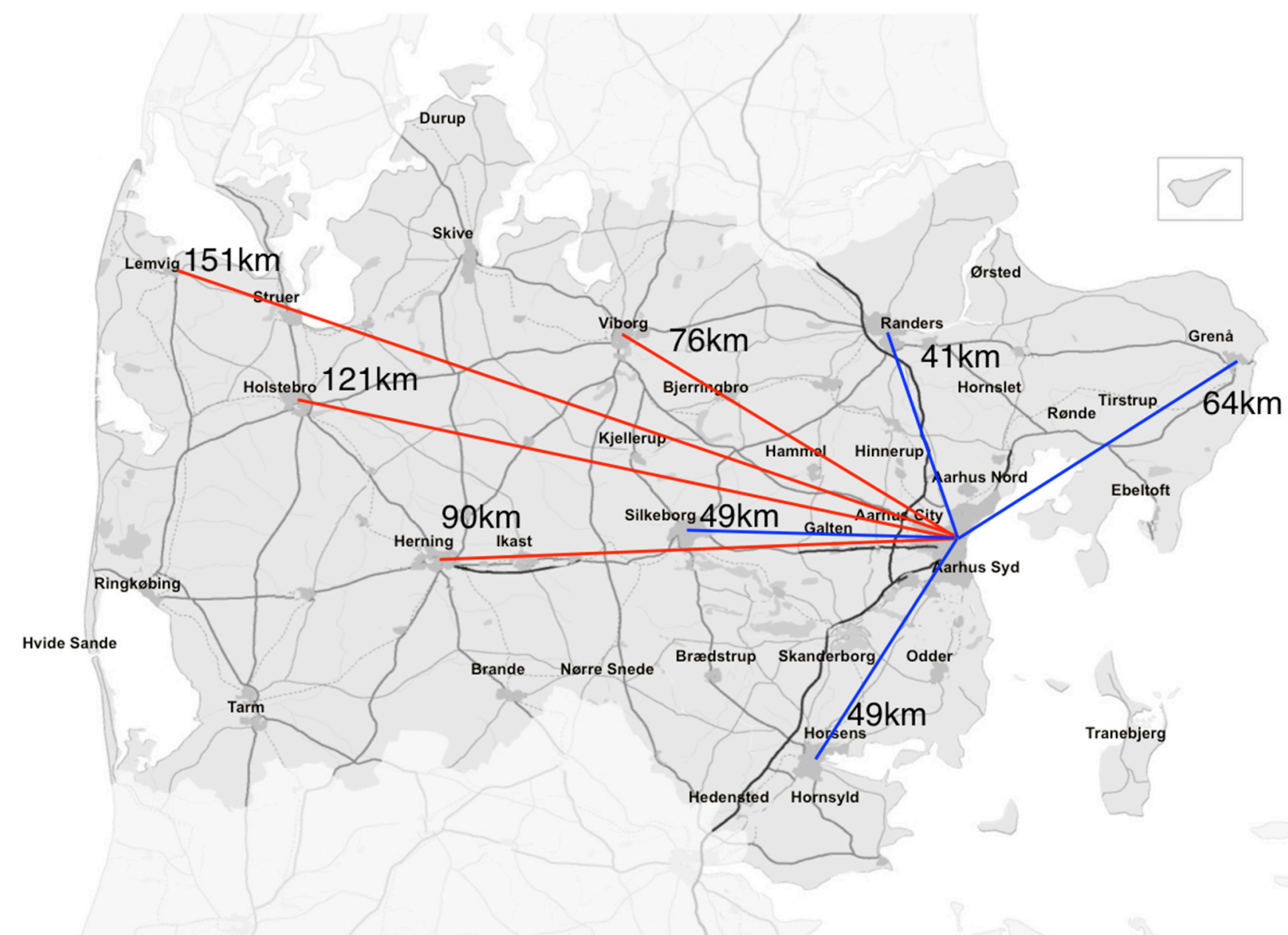

Figure 1 Map of Central Denmark Region showing the nine rapid response vehicle prehospital critical care teams (PHCCTs) connected to Aarhus University Hospital. The shortest possible travel distances by car have been estimated with Google Maps. ${ }^{31}$ The blue lines represent routes from PHCCTs to Aarhus University Hospital, where the proportion of diverted patients are larger by a factor of 3 , compared with the routes marked with red.

contributing to variations in the number and proportion of PHCCT-diverted patients from the different PHCCTs.

In Aarhus, the university hospital consists of five clinical centres at different locations, each housing several highly specialised departments. Given that we only have access to information regarding the clinical centre the patients are transported to and not the specific department, we excluded all patients who may have been transported to the ED by default or may have fulfilled the criteria for a predefined fast-track protocol. This exclusion may explain the apparent low incidence of patient-tailored triage within the city of Aarhus.

\section{Comparison of the number and proportion of PHCCT-diverted patients from PHCCTs located at a distance more than $70 \mathrm{~km}$ from Aarhus University Hospital with those located less than $70 \mathrm{~km}$ away}

The number of PHCCT patient contacts is unsurprisingly lower in the rural parts of the Central Denmark Region due to the smaller population. A more liberal HEMS dispatch to the western and more rural parts of the Central Denmark Region could explain why the proportion of PHCCT-diverted patients is reduced on average by approximately a factor of $3,{ }^{29}$ comparing the PHCCTs with a travel distance of more than $70 \mathrm{~km}$ to the university hospital with those less than $70 \mathrm{~km}$ away (table 1 and figure $1^{31}$ ). Furthermore, the PHCCTs may occasionally consider the travel time to Aarhus University Hospital from more rural areas to be too long due to the patient's condition and the need for urgent in-hospital treatment, for example, damage control surgery.

\section{Distribution of PHCCT-diverted patients sorted by prehospital diagnosis}

According to the European Emergency Data Project, five critical conditions, which are defined as the 'first hour quintet', are both life-threatening and of great economic importance. The 'first hour quintet' consists of cardiac arrest, respiratory failure, severe trauma, chest pain (including acute coronary syndrome) and stroke. These conditions represent typical emergency patients for which prehospital critical care can have a crucial impact. ${ }^{19}$ Approximately 1200/100 000 inhabitants per year in the Capital Region of Denmark, the Central Denmark Region and the Region of Southern Denmark presented to the EMS with one of these five conditions over a 6-month period in $2011 .^{29}$ As regional fast-track protocols cover the triage of patients with suspected acute coronary 
Table 2 Distribution of PHCCT-diverted patients among the 10 most frequent prehospital diagnoses

\begin{tabular}{|c|c|c|c|}
\hline \multirow[b]{2}{*}{ Rank } & \multicolumn{2}{|c|}{ Prehospital diagnosis } & \multirow[b]{2}{*}{ n (\%) } \\
\hline & Code & Description & \\
\hline 1 & DI460 & $\begin{array}{l}\text { Resuscitated from cardiac } \\
\text { arrest }\end{array}$ & $143(14)$ \\
\hline 2 & DZ041 & $\begin{array}{l}\text { Observation and } \\
\text { treatment following road } \\
\text { traffic accident }\end{array}$ & $105(11)$ \\
\hline 3 & DZ03/DZ039 & $\begin{array}{l}\text { Observation and } \\
\text { treatment after a non- } \\
\text { specified condition }\end{array}$ & $78(8)$ \\
\hline 4 & DI619 & Cerebral haemorrhage & $65(7)$ \\
\hline 5 & DR568D & Convulsions, unspecified & $58(6)$ \\
\hline 6 & DZ043 & $\begin{array}{l}\text { Observation and } \\
\text { treatment following other } \\
\text { accidents }\end{array}$ & $48(5)$ \\
\hline 7 & DI713/DI718 & $\begin{array}{l}\text { Dissecting/ruptured aortic } \\
\text { aneurysm }\end{array}$ & $47(5)$ \\
\hline 8 & DZ033 & $\begin{array}{l}\text { Observation and } \\
\text { treatment following injury } \\
\text { to the nervous system }\end{array}$ & $41(4)$ \\
\hline 9 & DS060 & Concussion/mild TBI & $41(4)$ \\
\hline 10 & DT079 & Polytrauma & $33(3)$ \\
\hline Total & & & $659(67)$ \\
\hline
\end{tabular}

$\%$, percentage of patients with the given diagnosis out of all PHCCT-diverted patients in the Central Denmark Region ( $n / 989) ; n$, number of PHCCT-diverted patients with given diagnosis.

PHCCT, prehospital critical care team; TBI, traumatic brain injury.

syndrome and stroke but not patients with cardiac arrest, respiratory failure or severe trauma, PHCCT-provided triage may be essential. Therefore, it may be considered appropriate that 'resuscitated from cardiac arrest' and 'multiple trauma' are among the most frequent diagnoses for PHCCT-diverted patients (table 3). Our data do not allow us to estimate the degree of overtriage and undertriage in our system.

\section{Distribution of PHCCT-diverted patients sorted by receiving hospital}

As the main referral centre and trauma centre, it is not surprising that the majority of PHCCT-diverted patients were transported to Aarhus University Hospital.

\section{Bias}

In the process of identifying all relevant diagnoses and receiving hospitals for the PHCCT-diverted patients in the Central Denmark Region, certain diagnoses or hospitals could have been overlooked. Thus, the overall proportion of PHCCT-diverted patients would probably be underestimated.

Our data set did not allow us to identify children under the age of 15 given that all patients were anonymous and lacked any specifying characteristics, that is, age. However, we know from service experience that the vast majority of children treated by the PHCCTs are triaged to the nearest paediatric department. Through the exclusion of patients admitted to the nearest hospital, we have also excluded most children. This exclusion criterion hopefully minimises the potential bias from not being able to exclude children under the age of 15 .

Given that trauma triage by the PHCCTs in children follows the same principles as in adults, the potential selection bias from not being able to exclude children suffering from major trauma is diminished. The low incidence of cardiovascular diseases among children, such as STEMI and stroke, is also important to bear in mind; however, this does not necessarily reduce this bias.

With regard to the secondary endpoint 'Number and proportion of PHCCT-diverted patients among patients treated by the different PHCCTs', there is likely some underestimation of the number and proportion of

Table 3 Distribution of PHCCT-diverted patients sorted by receiving hospital

\begin{tabular}{|c|c|c|c|c|c|c|c|}
\hline \multirow[b]{3}{*}{ РНСCT } & \multicolumn{6}{|c|}{ Receiving hospital } & \multirow[b]{3}{*}{ Total } \\
\hline & \multicolumn{4}{|c|}{ University hospital } & \multicolumn{2}{|c|}{ Regional hospital } & \\
\hline & AUH & AAUH & OUH & $\mathbf{R H}$ & Viborg & Holstebro & \\
\hline Lemvig & 23 & 1 & & 1 & 1 & & 26 \\
\hline Holstebro & 36 & 1 & & & 5 & & 42 \\
\hline Herning & 53 & & & 1 & 1 & & 55 \\
\hline Viborg & 52 & 4 & & 1 & & 3 & 60 \\
\hline Silkeborg & 171 & & & 1 & 7 & 1 & 180 \\
\hline Randers & 153 & & & 2 & & & 155 \\
\hline Grenaa & 170 & 1 & 1 & & & & 172 \\
\hline Horsens & 233 & 3 & 2 & 8 & & & 246 \\
\hline Aarhus & 52 & & & 1 & & & 53 \\
\hline Total & 943 & 10 & 3 & 15 & 14 & 4 & 989 \\
\hline
\end{tabular}

The four university hospitals represent the Central, North, Southern, Zealand and Capital Regions, separately.

AAUH, Aalborg University Hospital; AUH, Aarhus University Hospital; OUH, Odense University Hospital; PHCCT, prehospital critical care team; RH, Rigshospitalet. 
diverted patients from the four most western PHCCTs due to more frequent HEMS dispatch.

The PHCCTs in Herning and Holstebro did not register patient contacts in the electronic prehospital patient database of the Central Denmark Region until June/July 2013. Thus, the total number of contacts and PHCCT-diverted patients in this study is underestimated. However, data obtained from the remaining study period should be representative with regard to the proportion of PHCCT-diverted patients in Herning and Holstebro, as there were no major structural changes of the EMS of the Central Denmark Region at that time.

\section{Perspective}

This study aims to elucidate an anaesthesiologist-staffed prehospital critical care services' impact on patient flow through the healthcare system. We were not able to identify any other studies describing this topic. The findings therefore provide new knowledge into the complex prehospital critical decision-making processes performed by PHCCTs. These findings may serve as a basis for further investigations into prehospital triage in our region and for other physician-staffed prehospital critical care services. Further studies are warranted to document the efficacy and precision of this triage.

\section{CONCLUSION}

Our results demonstrated that in 1 in 40 contacts, that is, approximately 500 times a year, the PHCCTs in the Central Denmark Region divert critically ill and injured patients not included in a predefined fast-track protocol directly to a specialised department, potentially reducing time to definitive care for these patients. There may be a potential for increased diversion of non-fast-track patients directly to specialised departments in the Central Denmark Region.

Acknowledgements We sincerely thank Dr Uffe Landsfeldt for extracting the raw data from the prehospital critical care database.

Contributors LR conceived the study. MLF and LR designed the study. LR identified all relevant diagnoses and receiving hospitals for each PHCCT representing a direct referral to specialised department, and defined the inclusion and exclusion criteria. MLF performed the literature review and the identification of eligible patients from the prehospital database. MLF and LR analysed and interpreted the data. MLF wrote the manuscript. LR reviewed the manuscript. Both authors have read and approved the final manuscript.

Funding The authors have not declared a specific grant for this research from any funding agency in the public, commercial or not-for-profit sectors.

Competing interests None declared.

Patient consent Not required.

Ethics approval Given that this study is a retrospective descriptive study using anonymised data and a database previously approved by the Danish Data Protection Agency, consent from the ethical committee was not needed according to Danish law. No individual patients can be identified in our results.

Provenance and peer review Not commissioned; externally peer reviewed.

Data sharing statement All data generated or analysed during this study are included in this published article and in the full data set available from the Figshare Repository (D0l: 10.6084/m9.figshare.5852967).
Author note MLF is working as a medical doctor at the Department of Thoracic Medicine at Haukeland University Hospital. The research was performed during MLF's fifth and sixth year as a medical student at Aarhus University. LR is the lead clinician for the Pre-hospital Critical Care Service in Aarhus and the HEMS physician with the Danish Air Ambulance. He is an associate professor of prehospital care at Aarhus University.

Open access This is an open access article distributed in accordance with the Creative Commons Attribution Non Commercial (CC BY-NC 4.0) license, which permits others to distribute, remix, adapt, build upon this work non-commercially, and license their derivative works on different terms, provided the original work is properly cited, appropriate credit is given, any changes made indicated, and the use is non-commercial. See: http://creativecommons.org/licenses/by-nc/4.0/.

\section{REFERENCES}

1. Fevang E, Lockey D, Thompson J, et al. The top five research priorities in physician-provided pre-hospital critical care: a consensus report from a European research collaboration. Scand J Trauma Resusc Emerg Med 2011;19:57.

2. Krüger AJ, Skogvoll E, Castrén M, et al. Scandinavian pre-hospital physician-manned Emergency Medical Services--same concept across borders? Resuscitation 2010;81:427-33.

3. De Luca G, Suryapranata H, Ottervanger JP, et al. Time delay to treatment and mortality in primary angioplasty for acute myocardial infarction: every minute of delay counts. Circulation 2004;109:1223-5.

4. Sørensen JT, Terkelsen CJ, Nørgaard BL, et al. Urban and rural implementation of pre-hospital diagnosis and direct referral for primary percutaneous coronary intervention in patients with acute ST-elevation myocardial infarction. Eur Heart J 2011;32:430-6.

5. Terkelsen CJ, Sørensen JT, Maeng M, et al. System delay and mortality among patients with STEMI treated with primary percutaneous coronary intervention. JAMA 2010;304:763.

6. Prabhakaran S, O'Neill K, Stein-Spencer L, et al. Prehospital triage to primary stroke centers and rate of stroke thrombolysis. JAMA Neurol 2013;70:1126-32.

7. Prabhakaran S, Ruff I, Bernstein RA. Acute stroke intervention: a systematic review. JAMA 2015;313:1451-62.

8. Marchese G, Prochazka B, Widimsky P. The importance of time: Time delays in acute stroke. Cor Vasa 2016;58:e225-32.

9. Brunetti ND, De Gennaro L, Correale M, et al. Pre-hospital electrocardiogram triage with telemedicine near halves time to treatment in STEMI: A meta-analysis and meta-regression analysis of non-randomized studies. Int J Cardiol 2017;232:5-11.

10. Fransen PS, Berkhemer OA, Lingsma HF, et al. Time to Reperfusion and Treatment Effect for Acute Ischemic Stroke: A Randomized Clinical Trial. JAMA Neurol 2016;73:190-6.

11. Rodrigues FB, Neves JB, Caldeira D, et al. Endovascular treatment versus medical care alone for ischaemic stroke: systematic review and meta-analysis. BMJ 2016;353:i1754.

12. Rognås L, Hansen TM, Kirkegaard $\mathrm{H}$, et al. Pre-hospital advanced airway management by experienced anaesthesiologists: a prospective descriptive study. Scand J Trauma Resusc Emerg Med 2013;21:58.

13. Gaarder C, Holtan A, Naess PA. Prehospital point-of-care monitoring and goal-directed therapy: Does it make a difference? J Trauma Acute Care Surg 2015;78(6 Suppl 1):S60-4.

14. Ryynänen OP, lirola T, Reitala J, et al. Is advanced life support better than basic life support in prehospital care? A systematic review. Scand J Trauma Resusc Emerg Med 2010;18:62.

15. El Sayed MJ, Zaghrini E. Prehospital emergency ultrasound: a review of current clinical applications, challenges, and future implications. Emerg Med Int 2013;2013:1-6.

16. Fischer M, Kamp J, Garcia-Castrillo Riesgo L, et al. Comparing emergency medical service systems -A project of the European Emergency Data (EED) Project. Resuscitation 2011;82:285-93.

17. Rudolph SS, Sørensen MK, Svane C, et al. Effect of prehospital ultrasound on clinical outcomes of non-trauma patients-A systematic review. Resuscitation 2014;85:21-30.

18. Mikkelsen S, Kruger AJ, Zwisler ST, et al. Outcome following physician supervised prehospital resuscitation: a retrospective study. BMJ Open 2015;5:e006167.

19. Krafft T, García Castrillo-Riesgo L, Edwards S, et al. European Emergency Data Project (EED Project): EMS data-based health surveillance system. Eur J Public Health 2003;13(3 Suppl):85-90.

20. Sampalis JS, Denis R, Fréchette $P$, et al. Direct transport to tertiary trauma centers versus transfer from lower level facilities: impact on 
mortality and morbidity among patients with major trauma. $J$ Trauma 1997;43:288-95. discussion 95-6.

21. Pickering A, Cooper K, Harnan S, et al. Impact of prehospital transfer strategies in major trauma and head injury: systematic review, metaanalysis, and recommendations for study design. J Trauma Acute Care Surg 2015;78:164-77.

22. MacKenzie EJ, Rivara FP, Jurkovich GJ, et al. A national evaluation of the effect of trauma-center care on mortality. N Engl J Med 2006;354:366-78.

23. Meisler R, Berlac PA. [Prehospital trauma triage before and after deployment of a physician-manned mobile emergency care unit in Nordsjaelland]. Ugeskr Laeger 2009;171:2548-52.

24. Burén LA, Daugaard M, Larsen JK, et al. Visitation by physicians did not improve triage in trauma patients. Dan Med J 2013;60:A4717.

25. Hesselfeldt R, Steinmetz J, Jans $\mathrm{H}$, et al. Impact of a physicianstaffed helicopter on a regional trauma system: a prospective, controlled, observational study. Acta Anaesthesiol Scand 2013;57:660-8.

26. Chesters A, Fenton R. The effect of the introduction of a regional major trauma network on triage decisions made by a physician- staffed helicopter emergency medical service. Eur J Emerg Med 2015;22:395-400.

27. Danmarks statistik - Statistikbanken - befolkningstal [Internet]. 2014 http://www.statistikbanken.dk/FOLK1 (Cited 2/7/2015).

28. Andersen MS, Carlsen HP, Christensen EF. Criteria-based emergency medical dispatch of ambulances fulfils goals. Danish medical bulletin 2011;58:A4336.

29. Andersen MS, Johnsen SP, Sørensen JN, et al. Implementing a nationwide criteria-based emergency medical dispatch system: a register-based follow-up study. Scand J Trauma Resusc Emerg Med 2013;21:53.

30. About the hospital - Aarhus University Hospital. [Internet. http:// www.en.auh.dk/about-the-hospital/organisation/ (Cited 12 Feb 2018).

31. Google Maps [Internet]. https://www.google.dk/maps/place/Central+ Denmark+Region/@56.2313561,9.4475109,8.88z/data=!4m5!3m4! 1s0x464c0f667b950075:0x300afc9aca85430!8m2!3d56.302139!4d9. 3027769?hl=en (Cited 1 Mar 2018). 